\title{
S-MIM: A Novel Radio Interface for Efficient Messaging Services over Satellite
}

\author{
Sandro Scalise and Cristina Párraga Niebla, DLR Institute of Communications and Navigation \\ Riccardo De Gaudenzi and Óscar Del Río Herrero, European Space Agency
}

Daniele Finocchiaro and Antonio Arcidiacono, Eutelsat S.A.

\begin{abstract}
This article presents a radio interface recently standardized by ETSI under the name of S-band Mobile Interactive Multimedia (S-MIM) and especially designed to provide ubiquitous messaging services over S-band GEO satellites using low-power terminals. Thanks to low terminal cost and high bandwidth efficiency, this standard allows the development of new satellite services, particularly for vehicular and machine-to machine applications. The S-MIM standard relies on a broadcasting radio interface such as DVB-SH or ETSI SDR in the forward link, and reuses 3 GPP W-CDMA technology properly adapted to the scope in the return link. Thanks to the use of spread spectrum ALOHA, terminals can access the channel in a totally asynchronous manner. The use of a packet-optimized iterative successive interference cancellation (iSIC) algorithm at the receiver allows exploiting the inherent power imbalance among terminals in order to boost the throughput with respect to conventional SSA systems. In addition, advanced packet transmission control techniques have been adopted to maximize the probability of successful packet reception in the challenging land mobile satellite channel. Finally, the S-MIM link layer provides efficient and reliable transport of IP datagrams over the forward and return link radio interfaces.
\end{abstract}

\section{INTRODUCTION}

S-band satellite mobile broadcast systems are intended for reception of audio, video, and multimedia content by handheld or vehicle-mounted mobile devices. Complementary terrestrial transmitters can improve reception (e.g., in dense urban areas). Several systems are in operation in different parts of the world, and others are planned [1, 2]. A low-rate return channel is a valuable extension for broadcast systems, enabling interactive multimedia applications as well as other mobile applications. Examples are voting or shopping in conjunction with a TV program. Return channels exist for cable television, satellite direct-to-home (DTH), and terrestrial broadcast networks. Inside the S-band, the $2 \mathrm{GHz}$ mobile satellite service (MSS) band is of particular interest for interactive multimedia, since it allows two-way transmission through an uplink and a downlink band. The same frequency band can also be used for terrestrial base stations that are complementary to the satellite, hereafter referred to as the complementary ground component (CGC). Besides interactive broadcasting, communication systems in the $\mathrm{S}$ band are ideally suited for car connectivity and more in general for machine-to-machine (M2M) type of applications, since such services are often based on the transmission of small amounts of information from/to remote sensors or mobile devices used to track specific events or monitor some automatic systems, such as industrial supervisory control and data acquisition (SCADA) systems, fleet management or containers tracking, public systems, such as automatic highway tollgates, traffic light controllers, or energy systems (e.g., current sensors in a solar panel array, water level sensors in a dam). In recent years, M2M communications have been set up over terrestrial wireless networks, first using simple GSM SMS, and later general packet radio service (GPRS) and high-speed packet data access (HSPDA) technologies. Integrated satellite/terrestrial systems in the S-band now provide an attractive alternative to purely terrestrial systems, since they can work with comparable terminal equipment (in terms of technology, power consumption, size, and cost) and very effective bandwidth cost. Moreover, the satellite component brings some additional advantages, such as the immediate coverage of very large regions. The intrinsic cross-border nature of satellite makes it very competitive when providing mobile or M2M services over a wide area. Additionally, the independent nature of a satellite infrastructure also represents a unique and attractive feature for service providers when looking for a 


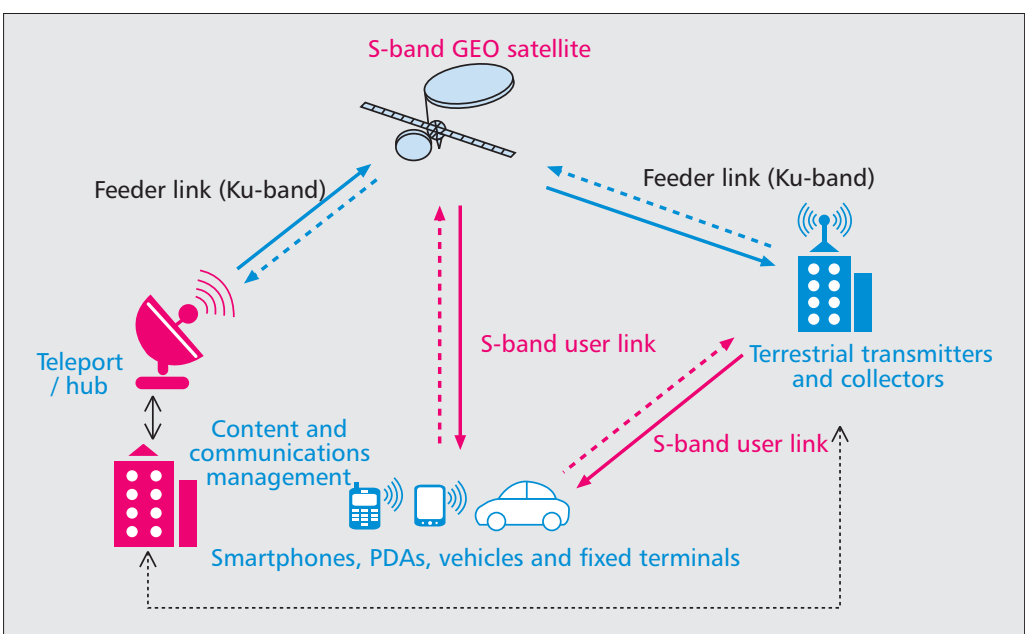

Figure 1. S-MIM system architecture. nized as follows. First, we describe the main technological innovation behind S-MIM; we then provide an overview of the system, and present the preliminary results of ongoing validation activities. Finally, our conclusions are presented.

\section{The Main Technical Challenges AND INNOVATION BEHIND S-MIM}

As depicted in Fig. 1, S-MIM is as an integrated satellite/terrestrial mobile system capable of providing interactive broadcast/multicast, data acquisition, and two-way messaging services to subscribers. Notably, the S-band allocation for pan-European MSS (60 MHz divided into 15 $\mathrm{MHz}$ in both uplink and downlink for each of the two licensed operators) allows the usage of this band for both satellite and terrestrial communications. Therefore, S-MIM relies on a bidirectional satellite component and a bidirectional CGC. The transparent S-band payload of a geostationary Earth orbit (GEO) satellite is assumed for the satellite component. However, non-GEO satellites are also compatible with this integrated system provided that Doppler precompensation countermeasures are put in place, and other frequency bands such as L-band, C-band, or $\mathrm{Ku} / \mathrm{Ka}$ band might be also considered upon suitable terminal and radio frequency (RF) front-end adaptations. Ku-band links are shown as examples of feeder links to the satellite S-band payload and the CGCs. In general, the feeder links to the S-band satellite payload and the CGCs are independent (i.e., the same or a different feeder link can be used, even in different frequency bands). Furthermore, the CGC feeder link can also be implemented by terrestrial networks.

There are a number of challenges to be tackled when designing a radio interface for a lowcost satellite mobile interactive system. The choice of the S-band frequency allocation next to the terrestrial Universal Mobile Telecommunications System (UMTS) allows having access to low-cost mass-market RF front-ends and in particular the high-power amplifier (HPA). To be able to exploit this synergy, the power requirements of the satellite user terminal (UT) shall be very similar to those of the terrestrial UMTS UT. This imposes a typical link margin of 3-6 $\mathrm{dB}$ for a UT with an EIRP of $0 \mathrm{dBW}$, useful bit rate of $5-10 \mathrm{~kb} / \mathrm{s}$, and satellite $\mathrm{G} / \mathrm{T}=8-9 \mathrm{~dB} / \mathrm{K}$ when the UT is operating in line-of-sight conditions and an additive white Gaussian noise (AWGN) channel. This link margin is clearly insufficient to counteract the depth of link shadowing that occurs in typical tree-shadowing and suburban environments of the land mobile satellite (LMS) channel model [6].

On the forward link, the S-MIM standard foresees that any satellite mobile broadcast radio access interface shall be used provided it fulfills the requirements defined in part 2 of [5]. If the digital video broadcast-satellite to handheld (DVB-SH) standard [7] is used, it is worth recalling that such a system largely counteracts the link interruptions by using channel coding combined with a long time interleaver at the physical or link layer level and admits the use of CGC to 

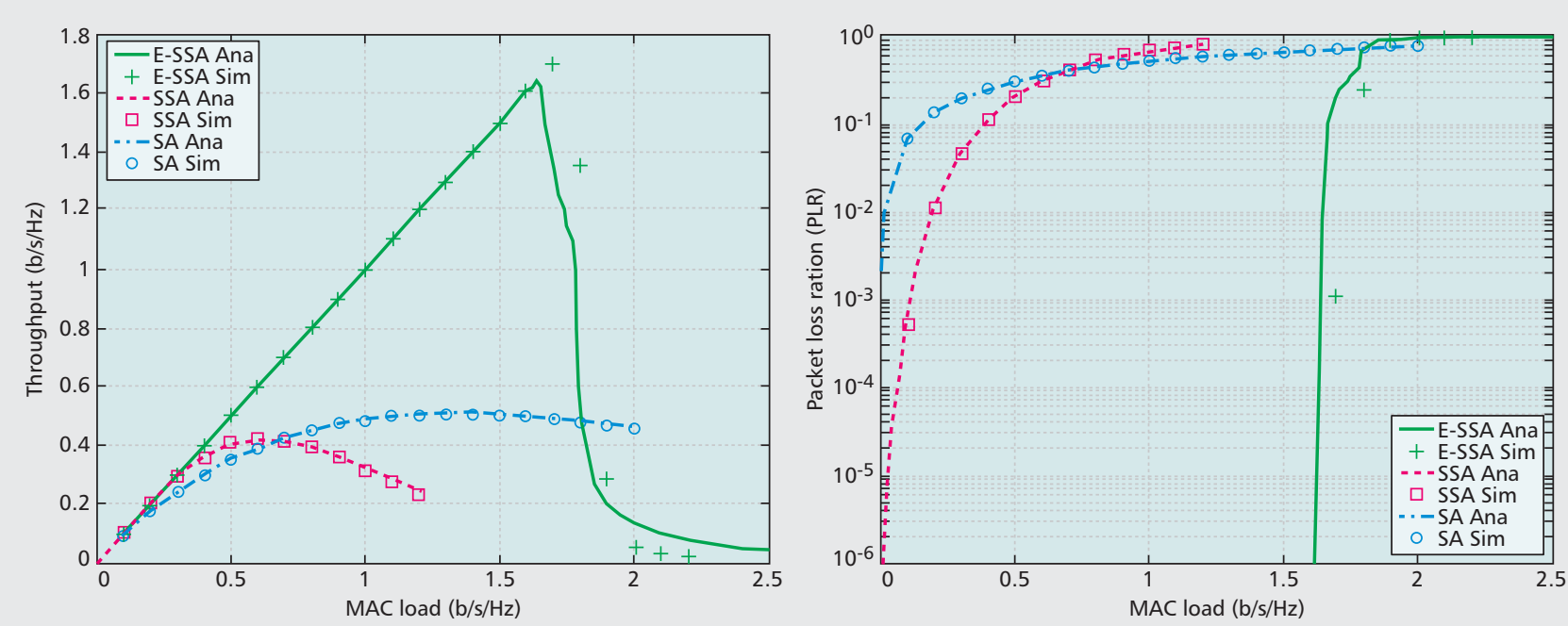

Figure 2. E-SSA random access theoretical and simulated performance in case of lognormal distributed power unbalance $(\sigma=2 d B)$ compared to conventional spread Aloha and slotted Aloha.

extend the satellite coverage in dense urban areas. In addition to state-of-the-art mobile broadcasting and multicasting capabilities, the most recent version ${ }^{2}$ of the DVB-SH standard features a low-latency interactive profile capability. This capability is important to support the SMIM messaging system signaling requirements. However, the DVB-SH low-latency profile (see Annex B of [7]) provides degraded performance compared to the nominal profile in terms of robustness to LMS channel impairments.

On the return link, the S-MIM radio interface is based on two non-exclusive options depending on the service required:

- Asynchronous random access using enhanced SSA (E-SSA) random access (RA) [12], described in [5, part3], for both satellite and CGC

- Synchronous demand assignment access using quasi-synchronous code-division multiple access (QS-CDMA), described in [5, part 4], for satellite only

The first radio interface is well suited for lowrate low-duty-cycle messaging services, whereas the second one is more suited for interactive services, including voice. The two access schemes might share the same spectrum or use different RF channels. The rest of this article focuses on the E-SSA RA, which is more innovative and relevant to the vehicular/telematics and M2M applications.

While in the forward link of a satellite mobile broadcasting system the LMS channel impairments can be largely counteracted by using coding combined with a long time interleaver at the physical or link layer level, as is the case in the DVB-SH system, the same is not applicable to the return link. To cope with the relevant channel impairments while keeping the UT EIRP at a level comparable to UMTS terminals, it is important to have a system whereby packet transmission is controlled to exploit the "good" or quasi-line of sight (LOS) channel conditions. The S-MIM UT observing the downlink channel estimates in real time the uplink link budget to transmit only when the conditions are sufficiently good, so as to maximize the probability of successfully delivering packets. On top of this, a uniform transmit power randomization is applied to achieve quasi-optimum sum rate throughput exploiting the i-SIC multi-user detection. At the same time, this transmit packet control policy avoids injecting harmful interference into the uplink shared channel. This approach may cause some transmission delay in case of persistent bad propagation conditions (e.g., shadowing/blockage), which is, however, compatible with the envisaged types of messaging and non-strictly real-time applications.

One of the key challenges in designing the SMIM standard has been to find an access scheme capable of providing high throughput with minimum overhead (i.e., signaling and retransmission) in the presence of sporadic traffic composed of small packets over the LMS channel. Classical RA schemes have been widely investigated in the literature and are known not to perform well in the satellite environment. RA techniques based on channel sensing commonly used in terrestrial networks cannot be exploited in satellite networks because of the large channel propagation delay. Open loop RA protocols such as slotted Aloha (SA) for time-division multiple access (TDMA) [8] and SSA for CDMA [9] are characterized by the fact that low packet collision probabilities (e.g. $<10^{-3}$ ) are achieved at very low loads. In case of SA, this is mainly due to the destructive effects of packet collisions, and for SSA to the high sensitivity to the packets power unbalance [12]. Operation in the high collision probability region is not practical in a satellite environment due to the high number of retransmissions needed yielding very high latencies. Therefore, their use today is mainly limited to initial network login and the transmission of capacity requests in contention minislots. In some cases, they are also used for the transmission of very small volumes of data, and combined free and demand assignment multiple access (CF-DAMA) [10] is usually the adopted
${ }^{2}$ Version 1.2.1 of DVBSH supports a low-latency profile that is required for sending acknowledgments, signaling information, and delay-sensitive data to the S-MIM user terminals. 


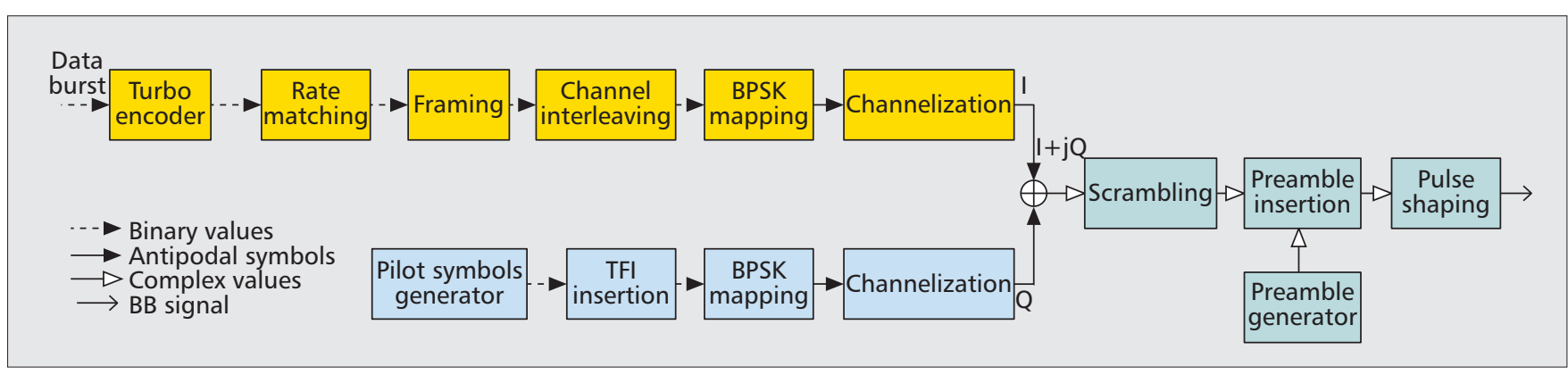

Figure 3. S-MIM transmitter functional block diagram (reproduced from [5]).

multiple access scheme for larger volumes or periodic transmissions of data. CF-DAMA has been shown in [10] to not be effective in an application scenario such as S-MIM.

\section{THE S-MIM RETURN LINK ASYNCHRONOUS RANDOM ACCESS ChANNEL}

The random access techniques adopted by SMIM is SSA. This technique was chosen because according to [10] it provides the highest spectral efficiency for a given user terminal EIRP ${ }^{3}$ in totally asynchronous mode, thus not requiring any control loop for keeping the user terminal synchronized as for slotted RA systems. Furthermore, thanks to the exploitation of optimized packet mode interference cancellation, E-SSA, different from conventional spread Aloha, is robust to the unavoidable received packets power unbalance. In addition, the throughput of E-SSA exploiting SIC can be optimized trying to approximate the optimum power distribution with ad hoc packet transmission control. As a result, the S-MIM E-SSA RA scheme achieves spectral efficiencies in excess of $1.5 \mathrm{~b} / \mathrm{s} / \mathrm{Hz}$ with packet loss ratio (PLR) less than $10^{-4}$ [12] (Fig. $2)$. This result represents three orders of magnitude higher throughput than conventional slotted Aloha, obtained thanks to the more complex signal processing implemented at the satellite station burst demodulator [12]. The demodulator complexity is readily achievable with modern field programmable gate arrays (FPGAs) or even using a software defined radio (SDR) approach as has been demonstrated by a Eutelsat funded development [13].

The 3GPP W-CDMA standard [11] provides a spread Aloha random access channel (RACH) that, with minor adaptations described in [5], has been exploited in the S-MIM standard to encompass the E-SSA RA scheme. The RACH is characterized by limited size data burst length, a collision risk, and the use of open loop power control. Terminals can access the channel in a totally asynchronous manner, provided they are locked to the forward link signal, they have received the system signaling tables distributed in the forward link, and the congestion control information distributed by the gateway enables transmission. Figure 3 shows a block diagram of a transmitter. The RACH is mapped on the physical data channel (PDCH) and sent on the
I-component. The related signaling information, that is, the actual PDCH configuration (e.g., data burst length, net data rate, chip rate), is signaled in an optional physical control channel $(\mathrm{PCCH})$, which can be sent in parallel to the data channel using the Q-component. A preamble is added to $\mathrm{I} / \mathrm{Q}$ multiplexing of the $\mathrm{PDCH}$ and $\mathrm{PCCH}$ to ensure correct detection at the gateway demodulator side even in the presence of a packet carrier frequency offset up to 2-3 $\mathrm{kHz}$. Given the requirement of implementing a hierarchical preamble (to reduce the complexity of the acquisition circuitry) composed of an outer code of length 96 symbols and an inner code of length 256 symbols for the case of 3.84 Mchips/s (or proportionally lower for lower chip rates), and the desire to actually implement multiple preamble codes by only changing the outer code (given the hardware simplification it provides), an extensive analysis on code families' characteristics led to the selection of a quaternary Golay code as the inner code and instead rely on a truncated Gold code family as outer codes. This allows implementing multiple preambles for reducing code collision (if required) while greatly simplifying the inner matched filter implementation. The advanced signal processing performed at the demodulator guarantees very high throughput, robustness to received packets power unbalance, totally asynchronous UT operation, and low UT EIRP requirement.

In order to improve system reliability, an automatic repeat request (ARQ) mechanism combined with a terminal rate control has been defined. The first mechanism provides retransmissions in case of packet losses in the E-SSA RA air interface, while the second allows the satellite gateway to tune the maximum rate at which terminals transmit in order to cope with congestion, which is critical in random access systems. The integration of these two mechanisms into one algorithm avoids retransmissions generated by the ARQ mechanism driving the system into congestion, so system stability in the access to the return link resources is preserved.

The selected ARQ is a cumulative stop-andwait (SW) mechanism applied at the link layer; that is, all link layer fragments corresponding to the same IP packet are transmitted consecutively, and a single acknowledgment (ACK) is sent by the gateway to notify the correct reception of all fragments. This scheme is very well suited for keeping the UT cost low due to its limited complexity and small buffer requirements; furthermore, the idle periods during the waiting time 
for an ACK are beneficial to spread the overall system load in the E-SSA channel. Other schemes that are known to be more efficient under full buffer assumptions, such as Go-Back$N$ (GNB) or Selective Repeat (SR), add significant complexity but do not outperform the SW mechanism in the service scenarios relevant to the S-MIM E-SSA low-rate messaging air interface. Furthermore, those more complex schemes require in-order delivery at the receiver and a sufficiently long sequence number, both conditions not guaranteed in S-MIM. The ARQ and rate control mechanism integrate by letting the satellite gateway tuning the allowed backoff between (re)transmissions together with the rest of ARQ parameters (waiting time for ACK, number of allowed retransmission) at the receiver through forward link dedicated signaling. This way, the satellite gateway instructs the UTs to transmit at lower rates when congestion is detected in the return link or allows them to transmit at higher rates when resources are underused.

S-MIM specifies the signaling format for such transmission control parameters and the procedure to be followed by the UTs. S-MIM allows three transmission modes: transparent mode (no ACK required), ACK mode (using hash values as ACKs to reduce the capacity requirement in the forward link), and uACK mode (using addressed ACKs) so that the most suitable transmission mode can be selected for each application according to its requirements. The method to estimate the load and the actual tuning of the transmission control parameters by the satellite gateway is implementation-dependent.

In addition to the above features, the satellite component can be complemented by a number of terrestrial "collectors." Different from the typical forward link gap fillers that are simply rebroadcasting on ground the satellite multiplex in dense urban areas, the collectors are mini-terrestrial gateways with S-MIM reception capability. The packets received from the UTs and demodulated at the collector are then conveyed to the satellite gateway by means of dedicated satellite or terrestrial links. The purpose of the collectors is twofold: a) to ensure good system performance in urban areas where the satellite availability is limited (satellite must be in LOS of the terminal), especially for applications that do not tolerate high delays; b) to offload the satellite from the traffic generated in high-density areas such as cities. This feature makes S-MIM inherently scalable, since the large footprint of the satellite could allow a very quick service rollout, whereas the progressive deployment of CGCs, which can all use the same portion of the S-band spectrum, will permit almost 100 percent service availability while avoiding congestion as the number of subscribers increases.

\section{EXPERIMENTAL RESULTS}

Thanks to a reference implementation of the SMIM physical layer based on SDR and carried out by the company MBI under Eutelsat specifications [13], it was possible to first test in the laboratory the behavior of the system, in particular,

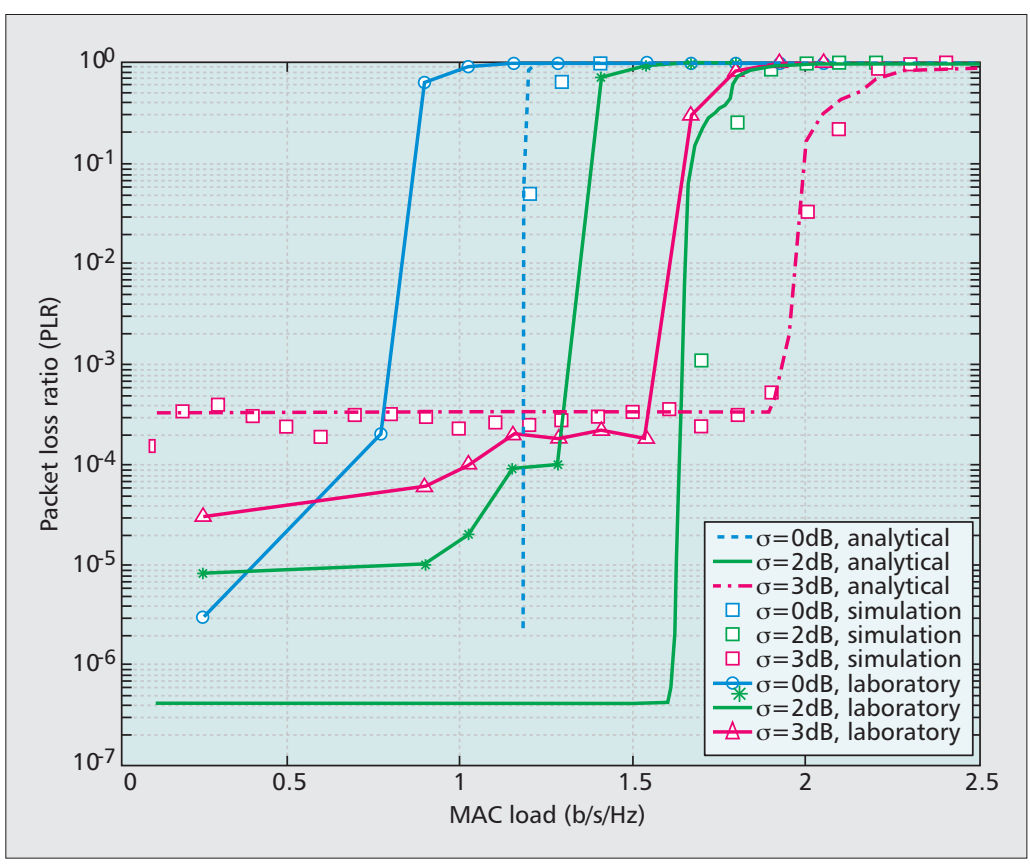

Figure 4. PLR vs. MAC loads and different values of the power imbalance standard deviation $(C / N=-16 \mathrm{~dB})$.

with respect to the packet-optimized SIC algorithm within the demodulator (described in [12]). According to the findings reported in [12] the gateway demodulator incoming packet power can be approximated as a lognormal distribution. In Fig. 4. some results obtained with the laboratory setup in terms of PLR at increasing medium access control (MAC) load are reported and compared to the theoretical and simulation results reported in [12] for different values of the packets' power unbalance assumed to be lognormally distributed with standard deviation $\sigma$. In general, it is known that a SIC-based MUD benefits from packet power unbalance. This is confirmed by analytical and simulation results reported in [12] and complemented by experimental laboratory findings. Measured performance are closer to theoretical and simulation performance when the lognormal standard deviation is larger. It is interesting to notice that up to a normalized MAC load of $1.7 \mathrm{~b} / \mathrm{s} / \mathrm{Hz}$, the system performance is optimized at $3 \mathrm{~dB}$ of standard deviation in terms of reached PLR. Above $1.7 \mathrm{~b} / \mathrm{s} / \mathrm{Hz}$, the standard deviation optimal value becomes higher. In reality it can be shown that a more optimum incoming packets random power distribution is represented by a uniform $\mathrm{dB}$ power distribution, which the S-MIM transmit packet control algorithm described in [5] attempts to achieve.

Within the EU project SafeTRIP [15], the first full implementation of the S-MIM standard has been developed including the physical and link layers in the terminal, and the satellite gateway. This setup is capable of demonstrating endto-end applications based on the S-MIM specification both in lab and field environments, the latter with real S-band satellite connectivity. Selected results gathered during the SafeTRIP laboratory and field trials are shown in Table 1, which provides trial results in terms of return 


The result is a system
allowing comple-
menting mobile
broadcast systems
with interactive
services, as well as
offering general
purpose messaging
services via hybrid
satellite/terrestrial
access. This system is
particularly well
suited for applica-
tions in the automo-
tive environment.

\begin{tabular}{|c|c|c|c|c|c|c|}
\hline \multicolumn{7}{|c|}{ Test configurations } \\
\hline Scenario ID & \multicolumn{2}{|c|}{ Environment } & \multicolumn{2}{|c|}{ Transmission mode } & \multicolumn{2}{|c|}{$\begin{array}{l}\text { Number of link layer } \\
\text { fragments per IP packet }\end{array}$} \\
\hline 1 & \multicolumn{2}{|l|}{ Field } & \multicolumn{2}{|l|}{ UACK } & \multicolumn{2}{|l|}{2} \\
\hline 2 & \multicolumn{2}{|c|}{ Laboratory } & \multicolumn{2}{|c|}{ Transparent } & \multicolumn{2}{|l|}{2} \\
\hline 3 & \multicolumn{2}{|c|}{ Laboratory } & \multicolumn{2}{|c|}{ Transparent } & \multicolumn{2}{|l|}{3} \\
\hline 4 & \multicolumn{2}{|c|}{ Laboratory } & \multicolumn{2}{|c|}{$\mathrm{ACK}, \mathrm{uACK}$, transparent } & \multicolumn{2}{|l|}{1,2} \\
\hline \multicolumn{7}{|c|}{ Test results } \\
\hline \multirow{2}{*}{ Scenario ID } & \multicolumn{2}{|c|}{$\begin{array}{l}\text { Return link QoS per- } \\
\text { formance }\end{array}$} & \multicolumn{2}{|c|}{ Processed packets } & \multicolumn{2}{|c|}{ Processed ACKs } \\
\hline & MLR & SDD (s) & Original & Retrans. & Total & Lost \\
\hline 1 & 0.0 & 3.5476 & 109 & 10 & 119 & 10 \\
\hline 2 & 0.0 & 3.0349 & 12814 & 0 & N.A. & N.A. \\
\hline 3 & 0.0 & 4.7938 & 1845 & 0 & N.A. & N.A. \\
\hline 4 & 0.0 & $\begin{array}{l}1.2407^{1} \\
3.0231^{2}\end{array}$ & 760 & 0 & 486 & 0 \\
\hline
\end{tabular}

Table 1. Safetrip link layer QoS tests' configuration and results.

link QoS including ARQ operation. The selected performance metrics are the message loss ratio (MLR) and service delivery delay (SDD). The MLR is the number of lost IP packets over the total transmitted IP packets; the SDD corresponds to averaging the total delay starting from the moment that the IP packet is put in the transmission queue until the moment that all link layer fragments of the same IP packet are correctly received. The impact of fragmentation and selected transmission mode in the QoS metrics can be observed in the results shown in Table 1, gathered in either laboratory or field environments. Clearly, SDD performance depends on the number of link layer fragments into which the IP packet needs to be fragmented to fit the S-MIM radio frame.

The correct operation of the ARQ mechanism was successfully tested. In scenario 1,10 packets needed to be retransmitted to achieve MLR $=0$. It is worth noting that after processing the results it was found out that the retransmissions were not required due to losses in the return link, but due to lost ACKs in the forward link. The results show that the implemented ESSA transmitter and receiver interact correctly to successfully deliver all IP packets.

\section{CONCLUSION}

The most important features of a novel bandwidth- and power-efficient radio interface for messaging services over satellite, recently stan- dardized by ETSI, have been presented in this article, highlighting on one hand the most relevant technical novelties entailed in the standard (notably the usage of an enhanced random access scheme which nevertheless keeps the terminal design extremely simple since it relies on advanced processing at the demodulator side), and on the other hand the most significant challenges that were addressed during the standardization effort. The result is a system allowing mobile broadcast systems to be complemented with interactive services, as well as offering general-purpose messaging services via hybrid satellite/terrestrial access. This system is particularly well suited for applications in the automotive environment (ranging from infotainment to driving assistance, telemetry, and the like) and the M2M scenario. For a complete description of the S-MIM system and all its features, please refer to the full system specifications [5].

\section{ACKNOWLEDGMENTS}

The authors would like to acknowledge all the participants to the DENISE project [14], jointly co-funded by ESA and Solaris Mobile Ltd, where most of S-MIM design and related standardization effort was carried out, as well as the SafeTRIP project co-funded by the European Commission [15]. A special mention goes to our colleague Gennaro Gallinaro for his decisive contribution to the S-MIM physical layer design. 


\section{REFERENCES}

[1] D. H. Layer, "Digital Radio Takes to the Road," IEEE Spectrum, vol. 38, no. 7, July 2001, pp. 40-46.

[2] C. Y. Yeun, "Mobile TV Technologies," Proc. 5th Int'l. Conf. on Info. and Commun. Technology, Cairo, Egypt, Dec. 16-18, 2007, pp. 2-9.

[3] I. M. Jacobs et al., "A Second Anniversary Operational Review of the OmniTracs: The First Two-Way Mobile KuBand Satellite Communications System," JPL, Caltech, Proc. 2nd Int'l. Mobile Satellite Conf., 1990, pp. 13-18.

[4] SAMOS Project Webpage: http://telecom.esa.int/telecom/www/object/index.cfm?fobjectid $=30896$

[5] ETSI TS 102 721, "Satellite Earth Stations and Systems (SES); Air Interface for S-band Mobile Interactive Multimedia (S-MIM)," v. 1.1.1.

[6] F. P. Fontan et al., "Statistical Modeling of the LMS Channel," IEEE Trans. Vehic. Technologies, vol. 50, no. 6, Nov. 2001, pp. 1549-67.

[7] ETSI EN 302 583, "Digital Video Broadcasting (DVB): Framing Structure, Channel Coding and Modulation for Satellite Services to Handheld Devices (SH) below 3 GHz," v. 1.2.1

[8] N. Abramson, "The Throughput of Packet Broadcasting Channels," IEEE Trans. Commun., vol. 25, no. 1, Jan. 1977, pp. 117-28.

[9] N. Abramson, "Spread Aloha CDMA Data Communications," U.S. Patent No. 5,537,397, July 16, 1996.

[10] R. De Gaudenzi and O. Del Rio Herrero, "Advances in Random Access Protocols for Satellite Networks," 2009 Int'l. Wksp. Satellite and Space Commun., Siena, Italy.

[11] 3GPP TS 25.213 v3.9.0, "Spreading and Modulation (FDD)," Release 1999, Jan. 2004.

[12] O. Del Río Herrero, R. De Gaudenzi, "High Efficiency Satellite Multiple Access Scheme for Machine-toMachine Communications," IEEE Trans. Aerospace and Electronic Systems, vol. 48, no. 4, Oct. 2012, pp. 2961-89.

[13] F. Basile and G. Mendola, "Satellite Hub Communication System GPU Based," GPU Technology Conf., 2012.

[14] DENISE Project: http://telecom.esa.int/DENISE.

[15] SafeTRIP Project: http://www.safetrip.eu.

\section{BIOGRAPHIES}

SANDRO SCALISE (Sandro.Scalise@dlr.de) graduated in electronic engineering specializing in telecommunications (with honors) from the University of Ferrara, Italy. In 2007, he received his Ph.D. (summa cum laude) from the University of Vigo, Spain. Since 2001, he is with the Institute of Communications and Navigation, DLR (German Aerospace Centre). Since July 2008, he has led the Satellite Networks Department, bearing responsibility for SatCom R\&D activities. He was responsible for S-MIM standardization.

CRISTINA PÁRRAga Niebla (Cristina.Parraga@dlr.de) graduated in telecommunications engineering from Universidad Politécnica de Catalunya (UPC), Barcelona, Spain, in July 2002. She has been with the Institute of Communications and Navigation, DLR, since 2002 as a research scientist. She led the design phase of the S-MIM system and contributed to the standardization effort.

RICCARDo DE GAUdEnzI (Riccardo.de.Gaudenzi@esa.int) received his D.Eng. degree (cum laude) in electronic engineering from the University of Pisa, Italy, in 1985 and his Ph.D. from the Technical University of Delft, The Netherlands, in 1999. In 1988 he joined ESA's Research and Technology Centre (ESTEC), Noordwijk, The Netherlands, where since 2005 he has been head of the RF Payload and Systems Division. He is co-inventor of the patent covering the fundamental principle of the E-SSA demodulator.

ÓsCAR DEL Río HERRERo (Oscar.Del.Rio.Herrero@esa.int) received his B.E. degree in telecommunications and $\mathrm{M} . \mathrm{E}$. degree in electronics from the University Ramon Llull, Barcelona, Spain, in 1992 and 1994, respectively. He joined ESTEC in 1996. He is a co-inventor of the patent covering the fundamental principle of the E-SSA demodulator.

DANIELE FINOCCHIARO (dfinocchiaro@eutelsat.fr) holds a Ph.D. in computer science from Scuola Normale Superiore in Pisa, Italy. He joined Eutelsat in 1999 where he works as projects coordinator in the Innovation and Deployment Department. He led the preliminary feasibility study for SMIM, and is currently involved in the development and deployment of S-MIM technology and its evolution.

ANTONIO ARCIDIACONO (aarcidiacono@eutelsat.fr) joined Eutelsat in 1990, where he is currently director of the Innovation and Deployment Department. With more than 20 years of experience in the space industry, he is a founding member of the DVB project and a member of the DVB Steering Board. He directed the technical development in the new field of S-band and initiated the activities for the creation of DVB-SH and ETSI S-MIM standards. 